\title{
Dependence of Diaminopurine Utilization on the Mutational Site of Purine Auxotrophy in Bacillus subtilis
}

\author{
II. Tracer Experiments \\ HAROLD T. SHIGEURA AND ARNOLD L. DEMAIN \\ Merck Sharp and Dohme Research Laboratories, Rahway, New Jersey 07065
}

Received for publication 24 November 1967

\begin{abstract}
Tracer experiments were carried out in an attempt to explain why guanineless auxotrophs can use diaminopurine as a guanine replacement but nonexacting purine auxotrophs cannot do so. Cell suspensions of the nonexacting purineless Bacillus subtilis MB-1356 incorporated more radioactivity from diaminopurine-2 $2{ }^{4} C$ into nucleic acid than did guanineless $B$. subtilis MB-1517. The radioactivity in MB-1356 ribonucleic acid (RNA) was distributed in both adenine and guanine nucleotides, thus eliminating the possibility that the deamination of diaminopurine to guanine occurred predominantly on the level of nucleoside di- or triphosphates. Strain MB-1517 incorporated adenine- $8-{ }^{14} \mathrm{C}$ into nucleic acids extremely poorly. This correlated with results obtained with cell-free extracts; strain MB-1517 showed much less adenosine monophosphate (AMP) pyrophosphorylase activity than did MB-1356. Likewise, guanineless MB-1517 converted diaminopurine to its nucleotide much more slowly than did the nonexacting purine auxotroph. The results indicated that the lack of growth of nonexacting auxotrophs on diaminopurine alone is due not to an inability to convert the analogue to nucleic acid adenine but to the greater capacity of the nonexacting auxotrophs to convert diaminopurine to its $5^{\prime}$-ribonucleotide. Presumably, this compound, or a coenzyme analogue produced from it, inhibits growth of mutants which cannot make AMP de novo and only when the medium is devoid of adenine.
\end{abstract}

The utilization of 2,6-diaminopurine by purine auxotrophs of Bacillus subtilis is dependent, in an interesting but as yet unexplained manner, on the site of the mutational lesion in the purine biosynthetic pathway. Both xanthineless and guanineless types of auxotrophs can grow on diaminopurine, thus showing that this synthetic purine can serve as a source of guanine in mutants that make their own adenosine-5'-monophosphate (AMP) by de novo synthesis. Specific adenineless mutants cannot use diaminopurine. This is understandable since diaminopurine compounds are not known to be directly converted to adenine derivatives, and, in such a mutant, the guanosine-5'-monophosphate (GMP) $\rightarrow$ AMP interconversion path would be blocked by the mutation. A puzzling response is shown by nonexacting purine auxotrophs which are blocked early in the de novo pathway but have an intact GMP $\rightarrow$ AMP interconversion cycle. These mutants grow on either adenine or guanine but for some unknown reason fail to utilize diaminopurine. Thus, we are faced with the paradox of diaminopurine being a precursor of guanine and guanine being a precursor of adenine but diaminopurine apparently failing to supply adenine.

In the preceding paper (10), several possible solutions to this question were rendered improbable by nutritional experiments. Two additional explanations remained to be tested experimentally. The first explanation postulated that the deamination of diaminopurine to guanine occurs at the level of ribonucleoside di- or triphosphates, thus bypassing the interconversion cycle which utilizes the monophosphates. In this case, diaminopurine would only serve as a source of guanine and could not provide adenine for nucleic acid synthesis. The alternative explanation postulated that, in addition to deamination of diaminopurine to guanine (at the level of a free base, a nucleoside, or a nucleoside monophosphate), there is a conversion of diaminopurine to its nucleoside monophosphate $(4,17,21)$ and this compound, or a coenzyme derivative (26), acts as a growth-inhibitory form 
of diaminopurine. Since the formation or action of such diaminopurine derivatives appears to be blocked by adenine derivatives $(21,23,26)$, only the nonexacting purine auxotrophs would be expected to be inhibited and the inhibition would occur only in the absence of natural purines. The xanthine and the guanine auxotrophs would grow on diaminopurine alone since the endogenously formed AMP would reverse the inhibition. The experiments to be described indicate that the second explanation is the correct one.

\section{MATERIALS AND METHODS}

Materials. 2,6-Diaminopurine-2-14C $(15.5 \mathrm{mc} /$ mmole) was obtained from Nuclear Equipment Chemical Corp. (Apollo, Pa.); adenine-8-14C (31.3 mc/ mmole) and guanine-8-14 $C(0.675 \mathrm{mc} / \mathrm{mmole})$ were purchased from Nuclear-Chicago Corp. (Des Plaines, III.). To test for purity of the radiochemicals, samples were applied to Whatman 3 MM paper and were developed overnight in a mixture of $95 \%$ ethyl alcohol and $1.0 \mathrm{M}$ ammonium acetate, $7: 3, \mathrm{v} / \mathrm{v}$ (solvent $\mathrm{A}$ ). The dried papers were examined for ultraviolet $a b-$ sorption under a mineralight lamp (Ultraviolet Products, Inc., San Gabriel, Calif.) and for radioactivity with a Packard liquid-scintillation counter (Packard Instrument Co., Inc., Downers Grove, Ill.). Each radioactive base showed only one ultravioletabsorbing spot, which corresponded to the respective standard and contained essentially all of the radioactivity applied to the paper.

Cultures. The $B$. subtilis mutants, the basal medium, and the methods of cultivation were described in the preceding paper (10).

Experiments with whole cells. B. subtilis strains, grown overnight in basal medium supplemented with $0.11 \mu$ mole of GMP per $\mathrm{ml}$, were harvested and the pellets were washed twice with $200 \mathrm{ml}$ of basal medium. The washed cells were suspended in the same medium and were used to examine the incorporation of diaminopurine, adenine, or guanine into acidinsoluble nucleic acids. The components of the incubation mixtures are given in the footnote of Table 1 . After $1.5 \mathrm{hr}, 9$ to $10 \mathrm{ml}$ of cold $10 \%$ trichloroacetic acid was added to the mixtures which were then centrifuged. The pellets were washed three times with cold $10 \%$ trichloroacetic acid and once each with $95 \%$ ethyl alcohol and $95 \%$ ethyl alcohol-ether $(3: 1)$, and then were plated on planchets and measured for radioactivity by use of a gas-flow counter. The results are expressed as millimicromoles of labeled base incorporated into acid-insoluble residue.

To study the incorporation of diaminopurine or guanine into the purine nucleotides of ribonucleic acid (RNA), a larger incubation mixture was used. The components of the incubation mixtures are given in the footnote of Table 2 . After $1.5 \mathrm{hr}$, the mixtures were treated with cold $10 \%$ trichloroacetic acid and were washed as described above. The acid-insoluble, lipid-free residues were mixed with $3 \mathrm{ml}$ of $0.3 \mathrm{~N} \mathrm{KOH}$ and were incubated at $30 \mathrm{C}$ for about $20 \mathrm{hr}$. The ribonucleotides were separated by means of Dowex-1- formate chromatography, were purified by adsorption on charcoal, and were measured for radioactivity (15). For further characterization, the purine nucleotides were applied to Whatman $3 \mathbf{M M}$ paper and the chromatograms were developed with solvent A. Both $2^{\prime}\left(3^{\prime}\right)$-AMP and $2^{\prime}\left(3^{\prime}\right)$-GMP gave single ultravioletabsorbing spots corresponding to AMP and GMP, respectively, by $R_{F}$ values and absorption spectra.

Experiments with cell-free extracts. B. subtilis MB1356 and MB-1517 were each grown overnight in 200 $\mathrm{ml}$ of GMP-supplemented basal medium $(0.11 \mu$ mole of GMP per $\mathrm{ml}$ ), and were harvested and washed as described above. Pellets of MB-1356 and MB-1517, wet weight 2.1 and $3.0 \mathrm{~g}$, respectively, were ground with three parts of alumina 301A in the cold for $3 \mathrm{~min}$. The ground pellets were mixed with $20 \mathrm{ml}$ of $0.05 \mathrm{M}$ phosphate buffer $(p H$ 7.2), and the mixture was centrifuged at $10,000 \times g$ for $15 \mathrm{~min}$. The clear supernatant fluids were used as the source of AMP pyrophosphorylase. The components of the reaction mixtures are given in the legend of Table 3. After incubation for a specified time, the mixtures were placed in a boiling-water bath for $3 \mathrm{~min}$, and were chilled and centrifuged. Portions of the clear supernatant fluids were applied on Whatman 3 MM paper and the chromatograms were developed with solvent A. Authentic compounds were also chromatographed as markers. The $R_{F}$ values of diaminopurine, adenine, guanine, AMP, and GMP were 0.46, 0.68, 0.55, 0.26, and 0.23 , respectively. These substances were also identified by their characteristic absorption spectra. In the above experiments, in which diaminopurine${ }^{2-14} C$ was used, a radioactive spot was also seen at $\boldsymbol{R}_{F}$ 0.12 . This material, like diaminopurine, showed a characteristic white fluorescence under a mineralight lamp, and its absorption spectrum was similar to that of diaminopurine. This material was assumed to be diaminopurine ribonucleoside monophosphate. The ultraviolet-absorbing spots were cut out, eluted with water, and measured for absorbance with a Cary recording spectrophotometer and for radioactivity with a Packard scintillation counter.

\section{RESUlts}

Incorporation of diaminopurine $-2-{ }^{14} \mathrm{C}$ into acidsoluble fraction. The first hypothesis to be considered was that the deamination of diaminopurine to guanine occurs at the ribonucleoside di- or triphosphate level and not at the stage of the free base, the nucleoside, or the nucleoside monophosphate. Although adenase and adenosine deaminase are known to deaminate diaminopurine and diaminopurine ribonucleoside, respectively $(8,13)$, it is possible that $B$. subtilis lacks these enzymes. The rationale for postulating such a situation is based on certain facts: (i) microorganisms and mammalian cells can convert diaminopurine or its ribonucleoside to the ribonucleoside di- and triphosphate forms (18. 21, 23); (ii) enzymes were described which deaminate adenosine triphosphate and adenosine diphosphate $(9,11,25)$; and (iii) certain known 
interconversions of purines and pyrimidine nucleotides proceed only at the diphosphate or triphosphate levels $(6,19)$.

To test this hypothesis, we first attempted to determine whether the nonexacting purine auxotroph, B. subtilis MB-1356, converts diaminopurine to the nucleoside di- and triphosphates. Four $\mathrm{ml}$ of packed cells were suspended in $\mathbf{1 6}$ $\mathrm{ml}$ of basal medium supplemented with $10 \mathrm{mg}$ of diaminopurine-2.14 $C(5 \mu \mathrm{c})$ and the suspension was shaken at $37 \mathrm{C}$. After $3 \mathrm{hr}$, the cell suspension was centrifuged; the pellet was mixed with $5 \mathrm{ml}$ of cold $0.4 \mathrm{M} \mathrm{HClO}_{4}$ and was centrifuged. After neutralization of the supernatant fluid with dilute $\mathrm{KOH}$, the ultraviolet-absorbing and radioactive components of the acid-soluble fraction were separated by means of Dowex-1-formate chromatography, as described elsewhere $(15,22)$. The chromatographic profile (not shown here) indicated that about $99 \%$ of the radioactivity in the acid-soluble fraction was present as unreacted diaminopurine. No radioactive material was present in the diphosphate and triphosphate zones. A trace of an ultraviolet-absorbing, radioactive substance was found in the nucleoside monophosphate region, but the amount was inadequate for characterization. We could only conclude that, under the experimental conditions used, diaminopurine was not phosphorylated to the di- and triphosphate level, although a trace amount of the monophosphate may have been formed. In these experiments, the acidinsoluble nucleic acids were not examined.

Incorporation of diaminopurine $-2 \cdot{ }^{14} \mathrm{C}$ into acidinsoluble material. Another test of the above hypothesis involved determining the site of incorporation of diaminopurine radioactivity into RNA in $B$. subtilis MB-1356, if diaminopurine radioactivity is incorporated at all. If deamination only occurs at the di- or triphosphate level, then only nucleic acid guanine should be labeled. Cell suspensions of $B$. subtilis MB-1356 were incubated with diaminopurine-2-14C and incorporation of this substance into acid-insoluble material was determined. Labeled adenine and guanine were used for comparison, as was the guanineless auxotroph, B. subtilis MB-1517. As shown in Table 1, the nonexacting strain MB-1356 (which cannot grow on diaminopurine alone) incorporated two to five times as much radioactivity from diaminopurine into an acid-insoluble form as did the guanineless mutant (which can grow on diaminopurine). Although strain MB-1356 incorporated about six times as much adenine or guanine as diaminopurine radioactivity in $1.5 \mathrm{hr}$, this could be the result of a permeability difference or of a slow rate of deamination. Guanineless strain MB-1517 also incorporated
TABLE 1. Incorporation of purines into acid-insoluble material by whole cells ${ }^{a}$

\begin{tabular}{|c|c|c|c|c|}
\hline \multirow[b]{2}{*}{ Expt } & \multirow[b]{2}{*}{${ }^{14} \mathrm{C}$ base } & \multirow[b]{2}{*}{$\begin{array}{l}\text { Amt } \\
\text { used }\end{array}$} & \multicolumn{2}{|c|}{$\begin{array}{c}\text { Amt (mumoles) } \\
\text { of }{ }^{14} \mathrm{C} \text { base } \\
\text { incorporated }\end{array}$} \\
\hline & & & $\begin{array}{c}\text { Strain } \\
\text { MB- } \\
\text { 1356, } \\
\text { (non- } \\
\text { exacting } \\
\text { purine- } \\
\text { less) }\end{array}$ & $\begin{array}{c}\text { Strain } \\
\text { MB- } \\
1517, \\
\text { (gua- } \\
\text { nine } \\
\text { less) }\end{array}$ \\
\hline \multirow{3}{*}{1} & & mumoles & & \\
\hline & Diaminopurine $-2-{ }^{14} C$ & 200 & 3.8 & 1.4 \\
\hline & Diaminopurine $-2-{ }^{14} C$ & 1,000 & 4.7 & 1.7 \\
\hline \multirow[t]{3}{*}{2} & Diaminopurine $-2-{ }^{14} C$ & 200 & 3.6 & 1.9 \\
\hline & Adenine $-8-{ }^{14} C$ & 200 & 22.3 & 0.5 \\
\hline & Guanine $-8-{ }^{14} \mathrm{C}$ & 200 & 23.8 & 15.5 \\
\hline \multirow[t]{3}{*}{3} & Diaminopurine-2 ${ }^{14} C$ & 200 & 11.3 & 2.3 \\
\hline & Adenine $-8-{ }^{14} C$ & 200 & 73.2 & 0.5 \\
\hline & Guanine $-8-{ }^{14} C$ & 200 & 68.4 & 11.1 \\
\hline
\end{tabular}

a The incubation mixture, in a total volume of $1.0 \mathrm{ml}$, contained the Bacillus subtilis cells suspended in basal medium containing diaminopurine $-2-{ }^{14} C$, adenine $-8-{ }^{14} C$, or guanine $-8-{ }^{14} C$. Vessels were shaken at $37 \mathrm{C}$ for $1.5 \mathrm{hr}$. OD at $660 \mathrm{~m} \mu$ of cell suspensions for experiments 1,2 , and 3 were 0.60 , 0.55 , and 0.70 , respectively.

guanine much faster than it incorporated diaminopurine. This finding correlates with our observation that the exponential rate of growth on diaminopurine is lower than that on guanine. Furthermore, when an equimolar concentration of unlabeled adenine or guanine was added to the diaminopurine-2-14 $C$ in experiments 2 and 3 (not shown in Table 1), the incorporation by both strains was diluted two- to threefold. Diaminopurine, on the other hand, did not markedly dilute the radioactivity incorporated from adenine- $8-{ }^{14} C$ or from guanine- $8-{ }^{14} C$. The most striking observation in Table 1 is the very low level of incorporation of adenine by guanineless strain MB-1517, as compared to that of strain MB-1356. This may be due to dilution of labeled AMP by endogenous AMP, inhibition of AMP formation by endogenous AMP, or a lack of AMP pyrophosphorylase. This problem was examined with cell-free extracts and is described below. On the basis of the results shown in Table 1 , it may be concluded that the lack of growth of strain MB-1356 on diaminopurine alone is not due to its inability to utilize the analogue for nucleic acid synthesis.

The distribution of radioactivity from diaminopurine- $2-{ }^{14} C$ into the ribonucleotides of RNA is shown in Table 2. Guanine- $-8{ }^{14} C$ was used for 
TABLE 2. Incorporation of diaminopurine or guanine into RNA nucleotides by whole cells ${ }^{a}$

\begin{tabular}{|c|c|c|c|}
\hline \multirow{2}{*}{ Mutant } & \multirow{2}{*}{${ }^{14} \mathrm{C}$ base } & \multicolumn{2}{|c|}{$\begin{array}{l}\text { Amt (m } m \text { moles) } \\
\text { of }{ }^{14} \mathrm{C} \text { base } \\
\text { incorporated into }\end{array}$} \\
\hline & & $\begin{array}{l}2^{\prime}\left(3^{\prime}\right)- \\
\text { AMP }\end{array}$ & $\begin{array}{l}2^{\prime}\left(3^{\prime}\right)- \\
\text { GMP }\end{array}$ \\
\hline \multirow{2}{*}{$\begin{array}{c}\text { Nonexacting } \\
\text { purineless } \\
\text { MB-1356 }\end{array}$} & $\begin{array}{l}\text { Diaminopurine- } \\
2-14 C\end{array}$ & 21.4 & 32.3 \\
\hline & Guanine $-8-{ }^{14} C$ & 54.2 & 92.0 \\
\hline \multirow[t]{2}{*}{$\begin{array}{c}\text { Guanineless } \\
\text { MB-1517 }\end{array}$} & $\begin{array}{l}\text { Diaminopurine- } \\
2-{ }^{14} C\end{array}$ & 0.02 & 19.0 \\
\hline & Guanine $-8-{ }^{14} C$ & 9.6 & 97.5 \\
\hline
\end{tabular}

a The incubation medium, in a total volume of $10.0 \mathrm{ml}$, contained Bacillus subtilis cells suspended in basal medium (OD at $660 \mathrm{~m}_{\mu}=1.4$ ) and 2.0 $\mu$ moles of ejther diaminopurine-2-14C $(446,500$ counts/min) or guanine- $8-^{14} C(1,130,000$ counts/min). The mixtures were placed in $125-\mathrm{ml}$ flasks and were shaken for $1.5 \mathrm{hr}$ at $37 \mathrm{C}$.

comparison. Both diaminopurine and guanine were extensively incorporated into both purine nucleotides of RNA by nonexacting purineless B. subtilis MB-1356. The ratios of incorporation into AMP and GMP are practically identical with both diaminopurine and guanine. This extensive conversion of diaminopurine into RNA adenine eliminates the possibility of deamination occurring solely at the level of nucleoside di- or triphosphate. It is also clear that the interconversion pathway of diaminopurine from GMP to AMP via IMP is not impaired in strain MB-1356. Some other mechanism must be found to explain the inability of MB-1356 to grow on diaminopurine alone.

Table 2 also shows that guanineless MB-1517 incorporates both diaminopurine and guanine predominantly into RNA guanine. This mutant, which can grow on diaminopurine alone, is able to convert the analogue to GMP but need not effect its further conversion to AMP since the latter nucleotide can be formed de novo. The known inhibition of GMP reductase by adenosine triphosphate (20) probably prevents the interconversion.

Conversion of free bases to ribonucleoside monophosphates. The second hypothesis to be considered assumes deamination of diaminopurine to guanine at the level of a free base, a nucleoside, or a nucleoside monophosphate; this is consistent with the data in Table 2. More important, it further elicits a second conversion of diaminopurine, a pyrophosphorylation to its nucleoside monophosphate $(4,17,21)$. This nucleotide or coenzyme derivative (26) is probably the biologically inhibitory form of diaminopurine. Since adenine compounds can antagonize such a conversion $(21,23,26)$, xanthine and guanine auxotrophs could grow on diaminopurine because of their ability to make AMP by de novo synthesis. Since the nonexacting strain MB-1356 cannot make AMP de novo, its growth would be inhibited on diaminopurine alone; it could only grow when a natural purine was also present. The existence of this possibility is indicated by the data of Table 1 . The most striking difference between the two auxotrophs was the marked inability of guanineless MB-1517 to incorporate adenine into nucleic acids. If this were due to the inactivity of AMP pyrophosphorylase, the conversion of diaminopurine to its nucleoside monophosphate would also be blocked, since diaminopurine also uses AMP pyrophosphorylase $(16,17,18,24)$.

Cell-free preparations of both auxotrophs were examined for the formation of mononucleotides from either adenine or diaminopurine in the presence of 5-phosphoribosylpyrophosphate (PRPP). Table 3 shows that the formation of AMP from adenine was considerably poorer for guanineless strain MB-1517 than for the nonexacting purine auxotroph. Figure 1 shows the marked difference in conversion of diaminopurine to its nucleotide in the two organisms; MB-1517 was again much poorer than MB-1356.

\section{Discussion}

The purpose of the work described in this paper and in the preceding one (10) was to explain the puzzling observation that, in $B$. subtilis and in other bacteria, diaminopurine can replace guanine for guanineless mutants but cannot replace guanine for nonexacting purine auxo-

TABle 3. Conversion of adenine to AMP by cell-free extracts ${ }^{a}$

\begin{tabular}{c|c|c}
\hline \multirow{2}{*}{ Protein in cell extract } & \multicolumn{2}{|c}{ Amt (mumoles) of AMP formed } \\
\cline { 2 - 3 } & $\begin{array}{c}\text { Strain MB-1356 } \\
\text { (nonexacting } \\
\text { purineless) }\end{array}$ & $\begin{array}{c}\text { Strain MB-1517 } \\
\text { (guanineless) }\end{array}$ \\
\hline$m g$ & 43.0 & 9.6 \\
0.33 & 136.0 & 18.6 \\
1.00 &
\end{tabular}

${ }^{a}$ Reaction mixture, in a total volume of 1.0 $\mathrm{ml}$, contained $1.0 \mu$ mole of adenine $-8{ }^{14} C, 1.0$ $\mu$ mole of 5-phosphoribosyl-pyrophosphate, 50

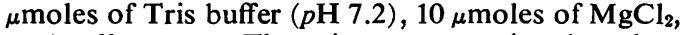
and cell extract. The mixtures were incubated at $37 \mathrm{C}$ for $20 \mathrm{~min}$. 


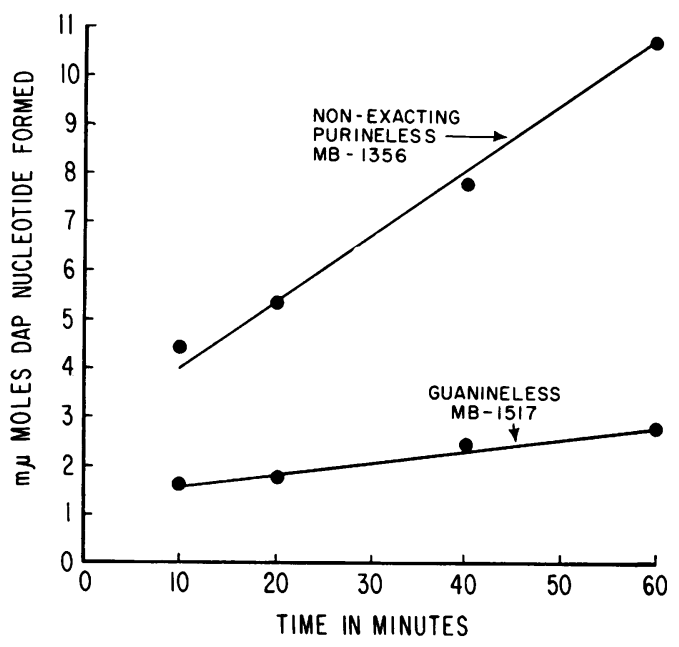

FIG. 1. Conversion of diaminopurine to its nucleotide by cell-free extracts. Reaction mixture, in a total volume of $1.0 \mathrm{ml}$, contained $1.0 \mu$ mole of diaminopurine-2-14 C, 1.0 mole of $P R P P, 50$ umoles of Tris buffer ( $\mathrm{pH} \mathrm{7.2),} 10$ umoles of $\mathrm{MgCl}_{2}$, and $1 \mathrm{mg}$ of protein as cell extract. Incubation was at $37 C$. No readings were made at zero-time.

trophs (blocked before IMP in the de novo pathway). Several hypotheses were examined which could explain this nutritional riddle, but only one hypothesis was supported by all of the nutritional and tracer experiments. This hypothesis postulates that nonexacting purineless auxotrophs fail to grow on diaminopurine as the sole purine source, since, in addition to deaminating diaminopurine to guanine (or diaminopurine riboside to guanosine), they convert diaminopurine to its nucleoside monophosphate via AMP pyrophosphorylase; diaminopurine riboside monophosphate or a coenzyme derivative is probably the biological inhibitory form of diaminopurine. Guanineless (and presumably also xanthineless mutants) can grow on diaminopurine because they are deficient in this enzyme activity and thus carry out the deamination reaction predominantly. The deficiency of AMP pyrophosphorylase is not thought to be a direct genetic effect of the original mutation but is probably due to feedback inhibition or repression by AMP or a derivative. Similar enzymes, which convert bases and ribonucleosides to ribonucleoside phosphates, were shown to be subject to feedback inhibition $(1,7,14)$, and there is no reason to suspect that repression does not also occur. That both inhibition and repression occur in vivo is suggested by the fact that the ratio of AMP pyrophosphorylase activities in cell-free extracts (where because of dilution, repression would be more important than inhibition) was only four- to sevenfold in favor of strain MB-1356 (Table 3), as compared to 44- to 135-fold (Table 1) when adenine incorporation into nucleic acids was tested in intact cells (where repression and inhibition would be important).

The utilization of diaminopurine for the synthesis of nucleic acid guanine and nucleic acid adenine, by both strains of $B$. subtilis, is consistent with previous results on other organisms which can also utilize exogenous guanine as a source of adenine $(2,3,12)$. Although diaminopurine is a precursor of both adenine and guanine purine nucleotides in $B$. subtilis, conversion to an inhibitory nucleotide form is probably antagonized only by adenine compounds. Previous studies on the biological activity of diaminopurine have established its nature as an adenine analogue. The triphosphate of diaminopurine replaces ATP in enzymatic reactions, the base and riboside are converted to the phosphorylated forms by the same enzymes which act on adenine compounds, various forms of diaminopurine are deaminated by the same enzymes which deaminate corresponding adenine forms, and the growth-inhibitory action of diaminopurine is reversed by adenine in many systems. In agreement with this concept is the finding of another study (10) that diaminopurine "spares" adenine to a much greater extent than it spares guanine for growth of the nonexacting strain MB-1356. The better growth on diaminopurine in the presence of adenine is evidently the result of inhibition of diaminopurine nucleotide formation by adenine. The limited protection by guanine is probably only effective to the extent of its conversion to AMP. In either case, when adenine compounds are depleted, MB-1356 is apparently forced to convert much diaminopurine to the nucleotide thereby resulting in cessation of growth. Guanineless MB-1517 does not need the protection of exogenous adenine since its rate of conversion of diaminopurine to the inhibitory nucleotide is evidently too low to inhibit cell growth.

Although it is generally thought that the active biological inhibitory form of diaminopurine is an analogue of an adenine-containing cofactor, such as the analogue of nicotinamide adenine dinucleotide formed by mammalian cells (26), it is clear that the ribonucleotide of diaminopurine is a key factor in growth inhibition (5). This was established in many systems where resistance to diaminopurine is associated with decreased formation of the nucleotide. It is tempting to postulate that wild-type $B$. subtilis is normally resistant to diaminopurine, owing to its great ability to produce purine nucleotides 
(and thus AMP) de novo (A. L. Demain, Progr. Ind. Microbiol., in press) and inhibition is only evident in mutants where the de novo pathway is blocked by mutation.

\section{ACKNOWLEDGMENTS}

We thank Kalinka Shumanov, Barbara Zych, and Joyce Kohler for technical assistance.

\section{Literature Cited}

1. Anderson, E. P., AND R. W. Brockman. 1964. Feedback inhibition of uridine kinase by cytidine triphosphate and uridine triphosphate. Biochim. Biophys. Acta 91:380-386.

2. Balis, M. E., M. S. Brooke, G. B. Brown, AND B. Magasanik. 1956. The utilization of purines by purineless mutants of Aerobacter aerogenes. J. Biol. Chem. 219:917-926.

3. Balis, M. E., D. H. Levin, G. B. Brown, G. B. Elion, H. VANDERWERFF, AND G. H. HITCHINGS. 1952. The incorporation of exogenous purines into pentose nucleic acid by Lactobacillus casei. J. Biol. Chem. 196:729-747.

4. Blair, D. G. R. 1967. Adenylate and 2,6-diaminopurine ribonucleotide pyrophosphorylase activities of $\mathbf{L}$ cells. Can. J. Biochem. Physiol. 45:435-448.

5. Blair, D. G. R., AND A. D. Hall. 1965. The metabolism of 2,6-diaminopurine by diaminopurine-sensitive and diaminopurine-resistant L-strain mouse fibroblasts. Can. J. Biochem. Physiol. 43:1857-1878.

6. Blakely, R. L. 1965. Cobamides and ribonucleotide reduction. I. Cobamide stimulation of ribonucleotide reduction in extracts of Lactobacillus leichmanii. J. Biol. Chem. 240: 2173-2180.

7. Bresnick, E., and R. J. Karjala. 1964. Endproduct inhibition of thymidine kinase activity in normal and leukemic human leukocytes. Cancer Res. 24:841-846.

8. Chilson, O. P., AND J. R. Fisher. 1963. Some comparative studies of calf and chicken adenosine deaminase. Arch. Biochem. Biophys. 102:77-85.

9. Chung, S. T., And K. Aida. 1967. Purification and properties of ATP deaminase from Microsporium audouini. J. Biochem. (Tokyo) 61:1-9.

10. Demain, A. L., and H. T. Shigeura. 1968. Dependence of diaminopurine utilization on the mutational site of purine auxotrophy in Bacillus subtilis. I. Nutritional experiments. J. Bacteriol. 95:565-571.

11. Deutsch, A., AND R. Nilsson. 1954. On the dephosphorylation and deamination of adenosine triphosphate by actomysin gel. Acta Chem. Scand. 8:1898-1906.

12. Hamilton, L. 1953. Utilization of purines for nucleic acid synthesis in chrysomonads and other organisms. Ann. N.Y. Acad. Sci. 56:961968.

13. Hartenstein, R. C., AND I. Fridovich. 1967. Adenine aminohydrolase. An investigation of specificity. J. Biol. Chem. 242:740-746.

14. Hatfield, D., AND J. B. WyngaARDen. 1964. 3-Ribosylpurines. I. Synthesis of (3-ribosyluric acid) 5'-phosphate and (3-ribosylxanthine) $5^{\prime}$-phosphate by a pyrimidine ribonucleotide pyrophosphorylase of beef erythrocytes. J. Biol. Chem. 239:2580-2586.

15. Hurlbert, R. B., H. Schmttz, A. F. Brumm, AND V. R. PotTer. 1954. Nucleotide metabolism. II. Chromatographic separation of acid-soluble nucleotides. J. Biol. Chem. 209: 23-39.

16. Kalle, G., AND J. S. Gots. 1961. Antagonisms between purines and purine analogues in auxotrophs of Salmonella typhimurium. J. Bacteriol. 81:331-337.

17. Kalle, G. P., and J. S. Gots. 1963. Genetic alteration of adenylic pyrophosphorylase in Salmonella. Science 142:680-681.

18. Kornderg, A., AND W. E. Pricer, Jr. 1951. Enzymatic phosphorylation of adenosine and 2,6-diaminopurine riboside. J. Biol. Chem. 193:481-495.

19. MagasaniK, B. 1962. Biosynthesis of purine and pyrimidine nucleotides, p. 295-334. In I. C. Gunsalus and R. Y. Stanier [ed.], The, bacteria. A treatise on structure and function, vol. 3. Academic Press, Inc., New York.

20. MAGer, J., AND B. MAGASANIK. 1960. Guanosine5 -phosphate reductase and its role in the interconversion of purine nucleotides. J. Biol. Chem. 235:1474-1478.

21. Remy, C. N., AND M. S. SMrth. 1957. Metabolism of diaminopurine: conversion to 5 '-phosphoribosyl-2-methylamino-6-aminopurine by enzymes of Escherichia coli. J. Biol. Chem. 228:325-338.

22. Shigeura, H. T., and C. N. Gordon. 1964. The effects of $3^{\prime}$-deoxyadenosine on the synthesis of ribonucleic acid. J. Biol. Chem. 240:806-810.

23. Tatibana, M., and H. Yoshikawa. 1962. Formation of 2-azadenine and 2,6-diaminopurine analogues of adenosine triphosphate in human erythrocytes. Biochim. Biophys. Acta 57:613615.

24. WAY, J. L., AND R. E. PARKs, JR. 1958. Enzymatic synthesis of 5 -phosphate nucleotides of purine analogs. J. Biol. Chem. 231 :467-480.

25. WeBster, H. L. 1953. Direct deamination of adenosine diphosphate by washed myofibrils. Nature 172:453-454.

26. Wheeler, G. P., AND B. J. Bowdon. 1966. Identification of analogues of nicotinamide adenine dinucleotide among the metabolites of 2,6-diaminopurine in mammalian cells. J. Biol. Chem. 241:1114-1121. 